\title{
TOPOLOGICAL QUASI *-ALGEBRAS AND THE TIME EVOLUTION OF $Q M_{\infty}$ SYSTEMS
}

\author{
FABIO BAGARELLO \\ Dipartimento di Metodi e Modelli Matematici, Facoltà di Ingegneria \\ Università di Palermo, Viale delle Scienze, I-90128 Palermo, Italy \\ E-mail: bagarell@unipa.it
}

\begin{abstract}
We give here a survey of some recent results on applications of topological quasi *-algebras to the analysis of the time evolution of quantum systems with infinitely many degrees of freedom.
\end{abstract}

1. Introduction. Let $\mathcal{S}$ be a microscopic physical system with finitely or infinitely many degrees of freedom. Its dynamical behavior is known (in principle) when the hamiltonian operator of $\mathcal{S}, H$, is given: by this we mean that, in the Schrödinger representation, the time evolution of the system is driven by the following (partial) differential equation:

$$
i \frac{d \Psi(\vec{r}, t)}{d t}=H \Psi(\vec{r}, t)
$$

while, in the Heisenberg representation, we have to solve this operatorial differential equation:

$$
\frac{d A(t)}{d t}=i[H, A(t)]
$$

Here $\Psi$ is the wave function of $\mathcal{S}$, while $A$ is any observable of the system.

For many physical systems (e.g. those with mean-field or long-range interactions), however, no operator $H$ can be rigorously defined. We only have a regularized operator, $H_{L}$, corresponding, for instance, to a finite volume version of $\mathcal{S}$. As a consequence of this fact, we can only know an approximate version of the dynamics of $\mathcal{S}$, obtained by solving

$$
i \frac{d \Psi_{L}(\vec{r}, t)}{d t}=H_{L} \Psi_{L}(\vec{r}, t) \quad \text { or } \quad \frac{d A_{L}(t)}{d t}=i\left[H_{L}, A_{L}(t)\right] .
$$

Then the following questions naturally arise: is it possible to remove the cutoff L? For which systems? And how? Is this limit related to the original (i.e. the infinitely extended) physical system?

2000 Mathematics Subject Classification: 81R15, 81V70.

The paper is in final form and no version of it will be published elsewhere. 
To deal with these questions an algebraic formulation of the dynamical description of quantum systems was originally proposed by Haag and Kastler, [HK]: its main ingredient is a $C^{*}$-algebra of the quasi-local observables, $\mathcal{A}$ : to each volume $V$ we associate the von Neumann algebra of the observables localized in $V, \mathcal{A}_{V}$. Then we take the union of all these algebras, $\mathcal{A}_{o}=\bigcup_{V} \mathcal{A}_{V}$, and we finally consider its completion $\mathcal{A}=\overline{\mathcal{A}}_{o} \|$ " , where \|\| is the $\mathrm{C}^{*}$-norm induced by $\mathcal{A}_{V}$.

However, several relevant models do not fit into this algebraic set-up: in fact, already for long-range interaction spin systems Robinson's constraint on the potential, $[\mathrm{BR}]$, is not satisfied and, as a consequence, the dynamics cannot be defined as a norm limit of the infrared cutoffed dynamics, since the time evolution of a strictly local variable may involve sequences of completely delocalized operators. The situation is even more difficult for continuous systems. Moreover, in most of $Q M_{\infty}$ systems, the hamiltonian $H$, when it exists, turns out to be an unbounded operator so that the use of $\mathrm{C}^{*}$-algebras as an underlying mathematical framework does not appear the most natural choice. To deal with these more realistic situations, two possible approaches have been developed during the years:

- on one hand, one can select a certain family of relevant states where the dynamics can be defined ([DS], [TW], [MS], [BM], etc...);

- on the other hand, one can enlarge the algebraic set-up: this has produced several extensions, as quasi *-algebras, [L], partial *-algebras, $[\mathrm{AK}]$ and $\mathrm{CQ}^{*}$-algebras, [BT3], among others.

It may be worth recalling that the problem of performing rigorously the thermodynamical limit of the time evolution of some local observables was the physical reason motivating for the introduction of quasi *-algebras in Lassner's treatment of the BCS model of superconductivity, [L], which is based on a model first introduced in [TW]. Here the physical system $\mathcal{S}$ is considered inside a box of finite volume $V$. Under this condition (fixed cutoff), we can safely write, since everything is well defined, the hamiltonian of the finite system $\mathcal{S}_{V}, H_{V}$, and its associated Heisenberg evolution,

$$
\alpha_{V}^{t}(X)=e^{i H_{V} t} X e^{-i H_{V} t}
$$

where $X$ is a local observable of the system. Here

$$
H_{V}=\frac{2 g}{|V|} \sum_{i, j \in V} \sigma_{i}^{-} \sigma_{j}^{+}+\epsilon \sum_{i \in V} \sigma_{i}^{3}=2 g|V| S_{V}^{-} S_{V}^{+}+\epsilon \sum_{i \in V} \sigma_{i}^{3},
$$

where $\sigma_{i}^{\alpha}$ is the $\alpha$-component of the $2 \times 2$ Pauli matrices localized at the lattice site $i \in V$, $g$ and $\epsilon$ are constants, and $S_{V}^{\alpha}=\frac{1}{|V|} \sum_{i \in V} \sigma_{i}^{\alpha}$.

It is now clear that, for finite $V, \alpha_{V}^{t}(X)$ belongs to the standard $\mathrm{C}^{*}$-algebra of the spin observables. However, to compute $\lim _{|V| \rightarrow \infty} \alpha_{V}^{t}(X)$, Lassner introduced the physical topology, $\tau$, different from the usual topologies on $\mathrm{C}^{*}$-algebras. This was necessary since $\lim _{|V| \rightarrow \infty} \alpha_{V}^{t}(X)$ does not exist, for generic $X$, in the uniform, strong or weak topologies. By means of this new topology, considering explicit estimates, Lassner proved that $\tau$ - $\lim _{|V| \rightarrow \infty} \alpha_{V}^{t}(X)$ exists, for any local observable $X$, and it belongs to the $\tau$-completion of the $C^{*}$-spin algebra, which turns out to be a topological quasi *-algebra. 
REMARK. As a technical tool, both [L] and [TW] introduced in their works an effective hamiltonian $H_{\text {eff }}$ which essentially shares with $H_{V}$ the property to reproduce the same equation of motion when $|V| \rightarrow \infty$ :

$$
i \frac{d \alpha_{V}^{t}(A)}{d t}=\left[H_{V}, \alpha_{V}^{t}(A)\right] \stackrel{|V| \rightarrow \infty}{\longrightarrow} i \frac{d \beta^{t}(A)}{d t}=\left[H_{e f f}, \beta^{t}(A)\right] .
$$

The relevance of $H_{e f f}$ is also that the spin-spin interaction present in $H_{V}$ is replaced by a simpler interaction between a spin and an external (fixed) magnetic field. It is easy to check that, whenever $H_{\text {eff }}$ does not depend explicitly on time, this last equation has the following solution: $\beta^{t}(A)=e^{i H_{e f f} t} A e^{-i H_{e f f} t}$. We will return to some features of the effective hamiltonians in Section 4.

In the past years we have extended several aspects of Lassner's procedure to get the rigorous definition of the algebraic dynamics, i.e. the time evolution of observables and/or states, for a general quantum physical system. We will see how in Sections 3 and 4, where we will always assume that a regularized hamiltonian $H_{L}$ ( $L$ is a model-depending cutoff) is given, which is bounded and self-adjoint in the Hilbert space $\mathcal{H}$ of the physical system. Then two possible situations may take place.

Suppose that $H_{L}$ converges to an operator $H$ : this is apparently the simplest situation. Of course we should specify the sense in which the convergence is understood. For each fixed $L$, we know the solution of the dynamical problem, i.e., we know the solution of the Heisenberg equation of motion

$$
i \frac{d \alpha_{L}^{t}(A)}{d t}=\left[H_{L}, \alpha_{L}^{t}(A)\right]
$$

This solution, $\alpha_{L}^{t}(A)=e^{i H_{L} t} A e^{-i H_{L} t}$, if $H_{L}$ does not depend explicitly on $t$, is called the regularized dynamics of the system. Then it makes sense to ask whether $\alpha_{L}^{t}(A)$ converges, possibly in the same sense as $H_{L}$ converges to $H$, to the solution $\alpha^{t}(A)$ of the (formal) Heisenberg equation

$$
i \frac{d \alpha^{t}(A)}{d t}=\left[H, \alpha^{t}(A)\right] .
$$

It is worth stressing that even though $H$ is a well defined self-adjoint operator, it is, in general, unbounded. For this reason, since domain problems in general arise, we say the (2) is formal.

The second situation is when $H_{L}$ does not converge (in any reasonable topology). This is quite a common situation, typical of mean field models, so that it is quite relevant for concrete applications. In this case, instead of $\alpha_{L}^{t}$, it is convenient to consider the derivations

$$
\delta_{L}(A)=i\left[H_{L}, A\right]
$$

that give, at the infinitesimal level, the dynamics of the system, $[S]$, and to analyze the convergence of $\delta_{L}$, the properties of the limit $\delta$ (when it exists), and, in particular, its spatiality. Also, it is quite interesting to understand whether $\delta$ produces a time evolution $\alpha^{t}$ for the system and which is the relation between $\alpha^{t}$ and $\alpha_{L}^{t}$.

REMARK. The situation changes if the physical system $\mathcal{S}$ is open. In this case $\mathcal{S}$ interacts with a reservoir $\mathcal{R}$, so that the energy of $\mathcal{S}$ is not necessarily preserved and the time 
evolution is given by a one-parameter semigroup (and not a group!). In this case the cutoff is introduced not on the hamiltonian of $\mathcal{S}$ but on the (Lindblad) generator of the semigroup. A few examples are discussed in [BS, B3, B4].

2. Mathematical preliminaries. Let $\mathcal{A}$ be a vector space and $\mathcal{A}_{o}$ a *algebra contained in $\mathcal{A}$. We say that $\mathcal{A}$ is a quasi ${ }^{*}$-algebra over $\mathcal{A}_{o}$ if

(i) the right and left multiplications of an element of $\mathcal{A}$ by an element of $\mathcal{A}_{o}$ are always defined and linear;

(ii) an involution * (which extends the involution of $\mathcal{A}_{o}$ ) is defined in $\mathcal{A}$ with the property that $(A B)^{*}=B^{*} A^{*}$ whenever the multiplication is defined.

A quasi *-algebra $\left(\mathcal{A}, \mathcal{A}_{o}\right)$ is said to have a unit $\mathcal{I}$ if there exists an element $\mathcal{I} \in \mathcal{A}_{o}$ such that $A \mathcal{I}=\mathcal{I} A=A, \forall A \in \mathcal{A}$. Finally, the quasi *-algebra $\left(\mathcal{A}, \mathcal{A}_{o}\right)$ is said to be topological if $\mathcal{A}$ carries a locally convex topology $\xi$ such that (a) the involution is continuous and the multiplications are separately continuous; and (b) $\mathcal{A}_{o}$ is dense in $\mathcal{A}[\xi]$ (see, for instance, $[\mathrm{Tr}])$.

The following example of a topological quasi *-algebra of operators is quite important for concrete applications.

Let $\mathcal{H}$ be a Hilbert space and $N$ an unbounded, self-adjoint operator defined on a dense domain $D(N) \subset \mathcal{H}$.

We call $D\left(N^{k}\right)$ the domain of the operator $N^{k}, k \in \mathbb{N}$, and $\mathcal{D}:=D^{\infty}(N)=$ $\cap_{k \geq 0} D\left(N^{k}\right)$, which is still dense in $\mathcal{H}$. On $\mathcal{D}$ we define a topology $t$ as follows:

$$
\phi \in \mathcal{D} \rightarrow\|\phi\|_{n}:=\left\|N^{n} \phi\right\|, n \in \mathbb{N}_{0},
$$

where \|\| is the norm of $\mathcal{H} . \mathcal{L}^{+}(\mathcal{D})$ is the ${ }^{*}$-algebra of all the closable operators defined on $\mathcal{D}$ which, together with their adjoints, map $\mathcal{D}$ into itself. Moreover, calling $\mathcal{D}^{\prime}$ the conjugate dual space of $\mathcal{D}$, endowed with the strong dual topology $t^{\prime}$, we define the set $\mathcal{L}\left(\mathcal{D}, \mathcal{D}^{\prime}\right)$ of all the continuous linear maps from $\mathcal{D}[t]$ into $\mathcal{D}^{\prime}\left[t^{\prime}\right]$.

The topologies on $\mathcal{L}^{+}(\mathcal{D})$ and $\mathcal{L}\left(\mathcal{D}, \mathcal{D}^{\prime}\right)$ are introduced by means of the set $\mathcal{C}$ of all positive, bounded and continuous functions $f(x)$ on $\mathbb{R}_{+}$satisfying the condition $\sup _{x \geq 0} f(x) x^{k}<\infty, \forall k \in \mathbb{N}$. The seminorms of the topology $\tau$ on $\mathcal{L}^{+}(\mathcal{D})$ are

$$
X \in \mathcal{L}^{+}(\mathcal{D}) \rightarrow\|X\|^{f, k}:=\max \left\{\left\|f(N) X N^{k}\right\|,\left\|N^{k} X f(N)\right\|\right\},
$$

where $k \geq 0$ and $f$ belongs to $\mathcal{C}$. Lassner proved in $[\mathrm{L}]$ that $\mathcal{L}^{+}(\mathcal{D})[\tau]$ is a complete locally convex topological *-algebra, with involution $X^{\dagger}:=\left.X^{*}\right|_{\mathcal{D}}$.

The seminorms of the uniform topology $\tau_{\mathcal{L}}$ on $\mathcal{L}\left(\mathcal{D}, \mathcal{D}^{\prime}\right)$ are defined by

$$
X \in \mathcal{L}\left(\mathcal{D}, \mathcal{D}^{\prime}\right) \rightarrow\|X\|^{f}:=\|f(N) X f(N)\|,
$$

where, again, $f$ belongs to $\mathcal{C} . \mathcal{L}\left(\mathcal{D}, \mathcal{D}^{\prime}\right)$, with the topology $\tau_{\mathcal{L}}$, is a topological quasi *-algebra over $\mathcal{L}^{+}(\mathcal{D})$.

The outcome of this example is that a single operator $N$ determines both an algebraic and a topological structure. Moreover, since the operator $N$ is usually defined in a natural way once the system $\mathcal{S}$ is given, [B1], it follows that the mathematical framework to be used to describe the time evolution of $\mathcal{S}$ is suggested by $\mathcal{S}$ itself. 
3. On the existence of $\lim _{L} \mathrm{e}^{i H_{L} t} A \mathrm{e}^{-i H_{L} t}$. In this section we will briefly discuss three different strategies which can be used in the analysis of the time evolution of $\mathcal{S}$. First of all we will consider some concrete models and we will use explicit estimates to show the existence of $\lim _{L} \mathrm{e}^{i H_{L} t} A \mathrm{e}^{-i H_{L} t}, A$ being an observable of $\mathcal{S}$. After, we will discuss a perturbative approach and finally we will consider some fixed point results.

3.1. Explicit estimates on $Q M_{\infty}$ models. We begin with the Almost Mean Field Ising Model, defined by the following finite volume hamiltonian:

$$
H_{V}=\frac{J}{|V|^{\gamma}} \sum_{i, j \in V} \sigma_{i}^{3} \sigma_{j}^{3}
$$

with $0<\gamma \leq 1$, [BT1]. Particularly relevant in the mathematical description of this model is the almost magnetization operator $S_{V}^{3}:=\frac{1}{|V|^{\gamma}} \sum_{p \in V} \sigma_{p}^{3}$. In fact, if $A$ is a local observable, its regularized time evolution $\alpha_{V}^{t}(A):=e^{i H_{V} t} A e^{-i H_{V} t}$ in general depends on $t, A$ and $S_{V}^{3}$.

For the reader's convenience we sketch now the construction given in [TW, L, BT1]. The model is defined on a lattice. To the lattice site $p$ we attach the Hilbert space $\mathbb{C}^{2}$ and the algebra of the $2 \times 2$ matrices generated by the identity matrix and the Pauli matrices $\sigma_{p}^{\alpha}$. The Hilbert space of the infinite lattice is $\mathcal{H}_{s p i n}=\otimes_{p \in \mathbb{Z}} \mathbb{C}_{p}^{2}$, and the algebra is the standard quasi local $\mathrm{C}^{*}$-spin algebra $\mathcal{A}_{s}$.

Let now $n=\left(n_{1}, n_{2}, n_{3}\right)$ be a unit vector in $\mathbb{R}^{3}$, and put $(\sigma \cdot n)=n_{1} \sigma^{1}+n_{2} \sigma^{2}+n_{3} \sigma^{3}$. The spectrum of $\sigma \cdot n$, is $\{1,-1\}$. We call $|n\rangle$ its unit eigenvector in $\mathbb{C}^{2}$ associated with 1 .

Let $\left(n, n^{1}, n^{2}\right)$ be an orthonormal basis of $\mathbb{R}^{3}$. We put $n^{ \pm}=\frac{1}{2}\left(n^{1} \pm i n^{2}\right)$ and, for $m=$ $0,1,|m, n\rangle=\left(\sigma \cdot n^{-}\right)^{m}|n\rangle$. We have $(\sigma \cdot n)|m, n\rangle=(-1)^{m}|m, n\rangle$. Repeating this procedure for each lattice site we get, starting with a sequence of vectors in $\mathbb{R}^{3},\{n\}:=\left\{n_{p}\right\}_{\{p \in \mathbb{Z}\}}$, a unit vector in $\mathcal{H}_{\text {spin }}$ defined as $|\{n\}\rangle=\otimes_{p}\left|n_{p}\right\rangle$. Furthermore, acting with the operators $\left(\sigma_{p} \cdot n_{p}^{-}\right)^{m_{p}}, p \in \mathbb{Z}$, on each $\left|n_{p}\right\rangle$, we get a new vector of $\mathcal{H}_{\text {spin }},|\{m\},\{n\}\rangle:=\otimes_{p}\left|m_{p}, n_{p}\right\rangle$. The set $\left\{|\{m\},\{n\}\rangle, m_{p}=0,1, \sum_{p} m_{p}<\infty\right\}$ forms an orthonormal basis in an Hilbert space $\{n\}$-depending, which we call $\mathcal{H}_{\{n\}}$. On this space we define the unbounded selfadjoint (number) operator $M_{\{n\}}$ by its action on the vectors of the basis

$$
M_{\{n\}}|\{m\},\{n\}\rangle=\left(1+\sum_{p} m_{p}\right)|\{m\},\{n\}\rangle .
$$

Of course $M_{\{n\}}$ depends on $\{n\}$, that is on the Hilbert space where the operator acts.

As it is discussed in [BT1], the sequence $\{n\}$ cannot be chosen arbitrarily. In fact, in order to analyze the thermodynamical limit of the model, we need to consider only those sequences such that $n_{p}^{1}=n_{p}^{2}=0$ for almost all $p \in \mathbb{Z}$ and $\lim _{|V| \rightarrow \infty} \frac{1}{|V|^{\gamma}} \sum_{p \in V} n_{p}^{3}$ exists in $\mathbb{R}$.

The algebraic setting is fixed by the operator $M_{\{n\}}$ as shown in the example of the previous section: first we put $\mathcal{D}_{\{n\}}=\bigcap_{k} \mathcal{D}\left(M_{\{n\}}^{k}\right)$. Then we introduce the algebra $\mathcal{L}^{\dagger}\left(\mathcal{D}_{\{n\}}\right)$, endowed with the quasi-uniform topology $\tau_{\{n\}}$. Finally we introduce a ${ }^{*}$-representation of the $\mathrm{C}^{*}$-spin algebra $\mathcal{A}_{s}$ on $\mathcal{L}^{\dagger}\left(\mathcal{D}_{\{n\}}\right)$ and a topology $\tau_{0}$ on $\mathcal{A}_{s}$ as follows:

$$
\pi_{\{n\}}\left(\sigma_{p}^{\alpha}\right)|\{m\},\{n\}\rangle=\sigma_{p}^{\alpha}\left|m_{p}, n_{p}\right\rangle \otimes\left(\prod_{p^{\prime} \neq p} \otimes\left|m_{p^{\prime}}, n_{p^{\prime}}\right\rangle\right) \quad(\alpha=1,2,3),
$$


and

$$
\|A\|_{\{n\}}^{f, k}:=\max \left\{\left\|M_{\{n\}}^{k} \pi_{\{n\}}(A) f\left(M_{\{n\}}\right)\right\|,\left\|f\left(M_{\{n\}}\right) \pi_{\{n\}}(A) M_{\{n\}}^{k}\right\|\right\},
$$

where $f$ belongs to $\mathcal{C}$ and $k \geq 0$. With these definitions, calling $\mathcal{A}$ the $\tau_{0}$-completion of $\mathcal{A}_{s}$, we proved in [BT1] that:

- $\left(\mathcal{A}\left[\tau_{0}\right], \mathcal{A}_{s}\right)$ is a topological quasi *-algebra;

- all the powers of the almost magnetization $S_{3}^{V}$ are $\tau_{0}$-converging in $\mathcal{A}$;

- the finite volume dynamics $\alpha_{V}^{t} \tau_{0}$-converges to a one-parameter group of automorphisms $\alpha^{t}$ of $\mathcal{A}$;

- $\alpha^{t}$ solves the $\tau_{0}$-limit of the finite volume Heisenberg equation of motion.

Another spin model which can be analyzed within the same algebraic framework is the almost mean field Heisenberg model,

$$
H_{V}=\frac{J}{|V|^{\gamma}} \sum_{i, j \in V} \sum_{\alpha=1}^{3} \sigma_{i}^{\alpha} \sigma_{j}^{\alpha},
$$

with $\frac{1}{2}<\gamma \leq 1$, see [BT2].

A different class of models that we have considered using the same approach involves free and interacting bosons. The formal hamiltonian $H$ for the one mode free bosons is simply the number operator $N=a^{\dagger} a, a$ and $a^{\dagger}$ being the annihilation and creation operators for the bosons. They satisfy the canonical commutation relation $\left[a, a^{\dagger}\right]=\mathcal{I}$. (More precisely, $N$ is the unique self-adjoint extension of the symmetric operator $a^{\dagger} a$.)

The construction of the topological quasi *-algebra is the same as before. Let $\mathcal{D}:=$ $D^{\infty}(N)=\cap_{k \geq 0} D\left(N^{k}\right)$. This set is dense in the Fock-Hilbert space $\mathcal{H}$ constructed in the standard way. Starting from $\mathcal{D}$ we can define the ${ }^{*}$-algebra $\mathcal{L}^{+}(\mathcal{D})$. It is clear that all powers of $a$ and $a^{\dagger}$ belong to this set. The topology in $\mathcal{L}^{+}(\mathcal{D})$ is the usual quasi-uniform topology:

$$
X \in \mathcal{L}^{+}(\mathcal{D}) \rightarrow\|X\|^{f, k}:=\max \left\{\left\|f(N) X N^{k}\right\|,\left\|N^{k} X f(N)\right\|\right\},
$$

where $f \in \mathcal{C}$ and $k \geq 0$. We know that $\mathcal{L}^{+}(\mathcal{D})[\tau]$ is a complete locally convex topological *-algebra.

Let $\mathcal{E}_{l}$ be the subspace of $\mathcal{H}$ generated by all the vectors which are proportional to $\left(a^{\dagger}\right)^{l} \Phi_{0}$. Let also $\mathcal{F}_{L}$ be the direct $\operatorname{sum} \mathcal{F}_{L}:=\mathcal{E}_{0} \oplus \mathcal{E}_{1} \oplus \ldots \oplus \mathcal{E}_{L}$. Finally, let $N=\sum_{l=0}^{\infty} l \Pi_{l}$ be the spectral decomposition of the number operator $N$. The operators $\Pi_{l}$ are projection operators, as are the operators $Q_{L}=\sum_{l=0}^{L} \Pi_{l}$. The following properties are obvious:

$$
\Pi_{k} \Pi_{l}=\delta_{k l} \Pi_{l}, \quad \Pi_{k}^{\dagger}=\Pi_{k} ; \quad Q_{L} Q_{M}=Q_{L}, \quad \text { if } L \leq M, \quad Q_{L}^{\dagger}=Q_{L} .
$$

It is clear that $\Pi_{k}: \mathcal{H} \rightarrow \mathcal{E}_{k}$, and $Q_{L}: \mathcal{H} \rightarrow \mathcal{F}_{L}$. The operator $Q_{L}$ is used to cut-off the hamiltonian, by replacing $a$ with $a_{L}:=Q_{L} a Q_{L}$. The regularized hamiltonian is simply $H_{L}=Q_{L} N Q_{L}=N Q_{L}$ and the related time evolution is $\alpha_{L}^{t}(X)=e^{i H_{L} t} X e^{-i H_{L} t}$. This occupation number cut-off produces a self-adjoint bounded operator $H_{L}$ and we have shown in [B1] that the limits of $\alpha_{L}^{t}\left(a^{n}\right)$ and $\alpha_{L}^{t}\left(\left(a^{\dagger}\right)^{n}\right)$ exist in $\mathcal{L}^{+}(\mathcal{D})[\tau]$ for all $n \in \mathbb{N}$. 
The same algebraic framework turns out to be useful also in the analysis of the thermodynamical limit of the interacting model described by the following formal hamiltonian:

$$
H_{V}=\frac{J}{|V|} \sum_{i, j \in V} \sigma_{i}^{3} \sigma_{j}^{3}+a^{\dagger} a+\gamma\left(a+a^{\dagger}\right) \sigma_{V}^{3},
$$

where $\sigma_{V}^{3}=\frac{1}{|V|} \sum_{i \in V} \sigma_{i}^{3}$. Here the algebra $\mathcal{L}^{+}(\mathcal{D})$ must be replaced by $\mathcal{A}=B\left(\mathcal{H}_{\text {spin }}\right) \otimes$ $\mathcal{L}^{+}(\mathcal{D})$. The topology on $\mathcal{A}, \tau_{\text {comp }}$, is generated by the following seminorms: $\|X A\|^{f, k, \Psi} \equiv$ $\|X\|^{f, k}\|A \Psi\|, X \in \mathcal{L}^{+}(\mathcal{D})$ and $A \in B\left(\mathcal{H}_{\text {spin }}\right)$. It is worthwhile to remind also that $\Psi$ cannot be a generic vector in $\mathcal{H}_{\text {spin }}$, but must belong to the set

$$
\mathcal{F}=\left\{\Psi \in \mathcal{H}_{\text {spin }}: \lim _{|V| \rightarrow \infty} \frac{1}{|V|} \sum_{p \in V} \sigma_{p}^{3} \Psi=\sigma_{\infty}^{3} \Psi,\left\|\sigma_{\infty}^{3}\right\| \leq 1\right\} .
$$

As before, the regularized hamiltonian is obtained by replacing $a$ with $a_{L}:=Q_{L} a Q_{L}$, so that the new hamiltonian $H_{V, L}$ depends on two, in principle, unrelated cutoffs. The existence of the limit of $\alpha_{V, L}^{t}(X)=e^{i H_{V, L} t} X e^{-i H_{V, L} t}$ is ensured by the following result, [B1]: the limit of $\alpha_{V, L}^{t}(a)$ for $|V|$ and $L$ both diverging exists in $\mathcal{A}[\tau]$. Moreover, if the two cutoffs satisfy the relation $|V|=L^{r}$, for a certain integer $r>1$, the same holds true also for $\alpha_{V, L}^{t}\left(\sigma_{\alpha}^{i}\right)$.

3.2. General results under given hypotheses on $H_{L}$. To begin with, we consider the simplest possible example in which a physical system is described by an unbounded, selfadjoint Hamiltonian $H_{0}$ on a Hilbert space $\mathcal{H}$; we assume $H_{0} \geq \mathcal{I}$; then $H_{0}$ has a spectral decomposition

$$
H_{0}=\int_{1}^{\infty} \lambda d E(\lambda)
$$

We define, for $L \geq 1$, the projectors

$$
Q_{L}^{0}=\int_{1}^{L} d E(\lambda)
$$

and, following a suggestion taken from the boson model, we introduce the regularized hamiltonian by:

$$
H_{L}=Q_{L}^{0} H_{0} Q_{L}^{0}
$$

Then, calling $\mathcal{D}=\mathcal{D}^{\infty}\left(H_{0}\right)$, it turns out that the operators $Q_{L}^{0}$ and $H_{L}$ are bounded operators in $\mathcal{B}(\mathcal{H})$ which belong to $\mathcal{L}^{\dagger}(\mathcal{D})$ and they commute with each other (and with $H_{0}$, in the usual sense).

This makes it quite easy to prove the following convergence properties, with $\tau$ the topology on $\mathcal{L}^{\dagger}(\mathcal{D})$ generated by the usual seminorms

$$
\mathcal{L}^{\dagger}(\mathcal{D}) \ni A \mapsto\|A\|^{f, k}=\max \left\{\left\|H_{0}^{k} A f\left(H_{0}\right)\right\|,\left\|f\left(H_{0}\right) A H_{0}^{k}\right\|\right\}:
$$

(c1) $H_{L} \rightarrow H_{0}$ with respect to the topology $\tau$,

(c2) $\mathrm{e}^{i t H_{L}} \rightarrow \mathrm{e}^{i t H_{0}}$ with respect to the topology $\tau$,

(c3) for each $A \in \mathcal{L}^{\dagger}(\mathcal{D}), \mathrm{e}^{i t H_{L}} A \mathrm{e}^{-i t H_{L}} \stackrel{\tau}{\rightarrow} \mathrm{e}^{i t H_{0}} A \mathrm{e}^{-i t H_{0}}$.

These results extend well known facts (i.e. the possibility of exponentiating a self-adjoint unbounded operators) using a different strategy. This may be more useful for physical 
applications, for instance when the hamiltonian is perturbed: let

$$
H=H_{0}+B
$$

where $B$ is regarded as a perturbation of the operator $H_{0}$. Practical reasons suggest to construct the cut-off starting from $H_{0}$, since $H_{0}$ can always be chosen in such a way that its spectral family $\{E(\lambda)\}$ can be explicitly obtained (this amounts to playing with the definitions of $H_{0}$ and $B$, once $H$ is given). Therefore we put

$$
H_{L}=Q_{L}^{0}\left(H_{0}+B\right) Q_{L}^{0}=H_{0} Q_{L}^{0}+Q_{L}^{0} B Q_{L}^{0} .
$$

The r.h.s. is well defined since $Q_{L}^{0} A Q_{L}^{0}$ is bounded for any $A \in \mathcal{L}^{\dagger}(\mathcal{D})$, [BT5]. We assume that

(a) $\mathcal{D}=\mathcal{D}^{\infty}\left(H_{0}\right)$;

(b) $D\left(H_{0}\right) \subseteq D(B)$ and $H=H_{0}+B$ is self-adjoint on $D\left(H_{0}\right)$;

(c) $\mathcal{D}^{\infty}\left(H_{0}\right)=\mathcal{D}^{\infty}(H)$.

Under these assumptions, we have:

LEMMA 1. (1) The topologies $t_{H_{0}}$ and $t_{H}$ are equivalent on $\mathcal{D}$;

(2) the topologies on $\mathcal{L}^{\dagger}(\mathcal{D})$ defined respectively by the set of seminorms

$$
\mathcal{L}^{\dagger}(\mathcal{D}) \in A \mapsto \max \left\{\left\|H_{0}^{k} A f\left(H_{0}\right)\right\|,\left\|f\left(H_{0}\right) A H_{0}^{k}\right\|\right\}, \quad f \in \mathcal{F}, k \in \mathbb{N},
$$

and

$$
\mathcal{L}^{\dagger}(\mathcal{D}) \in A \mapsto \max \left\{\left\|H^{k} A f(H)\right\|,\left\|f(H) A H^{k}\right\|\right\}, \quad f \in \mathcal{F}, k \in \mathbb{N},
$$

are equivalent.

Next we have the following results:

(1) For each $X \in \mathcal{L}^{\dagger}(\mathcal{D}), X=\tau$ - $\lim _{L \rightarrow \infty} Q_{L}^{0} X Q_{L}^{0}$;

(2) $\delta_{L}(A):=i\left[A, H_{L}\right]$ converges to $\delta(A):=i[A, H]$ with respect to the topology $\tau$;

(3) Concerning Condition (c) above, we have proved the following results:

Proposition 2. Let $B$ be a perturbation of $H_{0}$ such that $H:=H_{0}+B$ is self-adjoint on $D(H)=D\left(H_{0}\right)$. In order that

$$
\mathcal{D}^{\infty}(H)=\mathcal{D}^{\infty}\left(H_{0}\right)
$$

it is necessary and sufficient that the following conditions hold:

(i) $B: \mathcal{D}^{\infty}\left(H_{0}\right) \rightarrow \mathcal{D}^{\infty}\left(H_{0}\right)$;

(ii) $H$ is essentially self-adjoint in $\mathcal{D}^{\infty}\left(H_{0}\right)$;

(iii) the topologies $t_{H_{0}}$ and $t_{H}$ are equivalent on $\mathcal{D}^{\infty}\left(H_{0}\right)$,

Corollary 3. Let $B$ be a perturbation of $H_{0}$. Assume that $B$ satisfies the conditions:

(i) $<H_{0} f, B g>=<B f, H_{0} g>, \forall f, g \in \mathcal{D}^{\infty}\left(H_{0}\right)$;

(ii) $H$ is essentially self-adjoint in $\mathcal{D}^{\infty}\left(H_{0}\right)$;

(iii) $\left\|H_{0} f\right\| \leq\|H f\|, \quad \forall f \in \mathcal{D}^{\infty}\left(H_{0}\right)$.

Then $\mathcal{D}^{\infty}\left(H_{0}\right)=\mathcal{D}^{\infty}(H)$.

(4) Finally, going on with the analysis of the convergence of $e^{i H_{L} t}$, which ensures the convergence of the Schrödinger dynamics, we have the following result: 
Proposition 4. If there exists $T>0$ such that, for each $f \in \mathcal{F}, s \in \mathbb{N} \cup\{0\}$ there exists $M=M(T, f, s)$ such that

$$
\int_{0}^{t}\left\|f\left(H_{0}\right) \mathrm{e}^{i H_{L}\left(t-t^{\prime}\right)} H_{0}^{s}\right\| d t^{\prime}<M, \quad t \in[0, T]
$$

then

$$
\tau-\lim _{L \rightarrow \infty}\left(e^{i H_{L} t}-e^{i H t}\right)=0 .
$$

This assumption implies the existence of an uniform bound in $L$ : it is trivially verified if the perturbation $B$ commutes with $H_{0}$. Less trivial situations may be found in [BT5].

3.3. Fixed point results. This is an alternative procedure which eventually produces a rigorous definition of the dynamics of a (closed) physical system, see [B2].

Let $\mathcal{B}$ be a $\tau$-complete subspace of $\mathcal{L}^{+}(\mathcal{D})$ and $T$ a map from $\mathcal{B}$ into $\mathcal{B}$. We say that $T$ is a weak $\tau$ strict contraction over $\mathcal{B}$, briefly a $\operatorname{w} \tau \operatorname{sc}(\mathcal{B})$, if there exists a constant $c \in] 0,1[$ such that, for all $(h, k) \in \mathcal{C}_{N}:=(\mathcal{C}, \mathbb{N})$, there exists a pair $\left(h^{\prime}, k^{\prime}\right) \in \mathcal{C}_{N}$ satisfying

$$
\|T x-T y\|^{h, k} \leq c\|x-y\|^{h^{\prime}, k^{\prime}} \quad \forall x, y \in \mathcal{B} .
$$

In what follows we will consider equations of the form $T x=x, T$ being a $\mathrm{w} \tau \operatorname{sc}(\mathcal{B})$. The first step consists in introducing the following subset of $\mathcal{B}$ :

$$
\mathcal{B}_{L} \equiv\left\{x \in \mathcal{B}: \sup _{(h, k) \in \mathcal{C}_{N}}\|T x-x\|^{h, k} \leq L\right\},
$$

$L$ being a fixed positive real number.

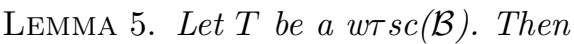

(a) if $T 0=0$ then any $x \in \mathcal{B}$ such that $\sup _{(h, k) \in \mathcal{C}_{N}}\|x\|^{h, k} \leq L_{1}$ belongs to $\mathcal{B}_{L}$ for $L \geq L_{1}(1+c)$;

(b) if $\|T 0\|^{h, k} \leq L_{2}$ for all $(h, k) \in \mathcal{C}_{N}$, then any $x \in \mathcal{B}$ such that $\sup _{(h, k) \in \mathcal{C}_{N}}\|x\|^{h, k} \leq$ $L_{1}$ belongs to $\mathcal{B}_{L}$ for $L \geq L_{1}(1+c)+L_{2}$;

(c) if $x \in \mathcal{B}_{L}$ then $T^{n} x \in \mathcal{B}_{L}$, for all $n \in \mathbb{N}$;

(d) $\mathcal{B}_{L}$ is $\tau$-complete;

(e) if $\mathcal{B}_{L}$ is not empty, then $T$ is a wrsc $\left(\mathcal{B}_{L}\right)$.

$\mathcal{B}_{L}$ is nonempty, see [B2]. The existence of a fixed point is ensured by the following Proposition:

Proposition 6. Let $T$ be a wisc(B). Then

(a) $\forall x_{0} \in \mathcal{B}_{L}$ the sequence $\left\{x_{n} \equiv T^{n} x_{0}\right\}_{n \geq 0}$ is $\tau$-Cauchy in $\mathcal{B}_{L}$. Its $\tau$-limit, $x \in \mathcal{B}_{L}$, is a fixed point of $T$;

(b) if $x_{0}, y_{0} \in \mathcal{B}_{L}$ satisfy the condition $\sup _{(h, k) \in \mathcal{C}_{N}}\left\|x_{0}-y_{0}\right\|^{h, k}<\infty$, then $\tau$ - $\lim _{n} T^{n} x_{0}$ $=\tau-\lim _{n} T^{n} y_{0}$.

For physical applications we need to consider the case in which these maps depend on an external parameter:

Let $I \subset \mathbb{R}$ be a set such that 0 is one of its accumulation points. A family of weak $\tau$ strict contractions $\left\{T_{\alpha}\right\}_{\alpha \in I}$ is said to be uniform if 
1) $T_{\alpha}: \mathcal{B} \rightarrow \mathcal{B} \forall \alpha \in I, \mathcal{B}$ being a $\tau$-complete subspace of $\mathcal{L}^{+}(\mathcal{D})$;

2) $\forall(h, k) \in \mathcal{C}_{N}$ and $\forall \alpha \in I$ there exist $\left(h^{\prime}, k^{\prime}\right) \in \mathcal{C}_{N}$, independent of $\alpha$, and $\left.c_{\alpha} \in\right] 0,1[$, independent of $(h, k)$, such that

$$
\left\|T_{\alpha} x-T_{\alpha} y\right\|^{h, k} \leq c_{\alpha}\|x-y\|^{h^{\prime}, k^{\prime}}, \quad \forall x, y \in \mathcal{B} ;
$$

3) $\left.c_{-} \equiv \lim _{\alpha \rightarrow 0} c_{\alpha} \in\right] 0,1[$.

We further say that the family $\left\{T_{\alpha}\right\}_{\alpha \in I}$ is $\tau$-strong Cauchy if, for all $(h, k) \in \mathcal{C}_{N}$ and $\forall y \in \mathcal{B}$,

$$
\left\|T_{\alpha} y-T_{\beta} y\right\|^{h, k} \stackrel{\alpha, \beta \rightarrow 0}{\longrightarrow} 0 .
$$

We set $\mathcal{B}_{L}^{(\alpha)} \equiv\left\{x \in \mathcal{B}: \sup _{(h, k) \in \mathcal{C}_{N}}\left\|T_{\alpha} x-x\right\|^{h, k} \leq L\right\}$.

Proposition 7. Let $\left\{T_{\alpha}\right\}_{\alpha \in I}$ be a $\tau$-strong Cauchy uniform family of $w \tau s c(\mathcal{B})$. Then

(1) There exists a $w \tau s c(\mathcal{B}), T$, which satisfies the following relations:

$$
\left\|T y-T_{\alpha} y\right\|^{h, k} \rightarrow 0 \quad \forall y \in \mathcal{B}, \forall(h, k) \in \mathcal{C}_{N}
$$

and

$$
\|T y-T z\|^{h, k} \leq c_{-}\|y-z\|^{h^{\prime}, k^{\prime}} \quad \forall y, z \in \mathcal{B},
$$

where $\left(h^{\prime}, k^{\prime}\right)$ are those of inequality (12).

(2) Let $\left\{x_{\alpha}\right\}_{\alpha \in I}$ be a family of fixed points of the net $\left\{T_{\alpha}\right\}_{\alpha \in I}: T_{\alpha} x_{\alpha}=x_{\alpha}, \forall \alpha \in I$. If $\left\{x_{\alpha}\right\}_{\alpha \in I}$ is a $\tau$-Cauchy net then, denoting by $x$ its $\tau$-limit in $\mathcal{B}, x$ is a fixed point of $T$.

(3) If the set $\cap_{\alpha \in I} \mathcal{B}_{L}^{(\alpha)}$ is not empty and if the following commutation rule holds:

$$
T_{\alpha}\left(T_{\beta} y\right)=T_{\beta}\left(T_{\alpha} y\right), \quad \forall \alpha, \beta \in I \text { and } \forall y \in \mathcal{B},
$$

then, writing

$$
x_{\alpha}=\tau \text { - } \lim _{n \rightarrow \infty} T_{\alpha}^{n} x^{0} \text {, where } x^{0} \in \cap_{\alpha \in I} \mathcal{B}_{L}^{(\alpha)},
$$

each $x_{\alpha}$ is a fixed point of $T_{\alpha}, T_{\alpha} x_{\alpha}=x_{\alpha}$ and $\left\{x_{\alpha}\right\}_{\alpha \in I}$ is a $\tau$-Cauchy net. Moreover $\tau-\lim _{\alpha \rightarrow 0} x_{\alpha}$ is a fixed point of $T$.

As an application we have proven in [B2] that, under certain technical assumptions, the time evolution of a given operator $x$,

$$
x_{\alpha}(t)=x+i \int_{0}^{t} d s\left[H_{\alpha}, x_{\alpha}(s)\right],
$$

is associated with a uniform family of $\mathrm{w} \tau \operatorname{sc}\left(\mathcal{L}^{+}\right),\left\{U_{\alpha}\right\}$, which is also $\tau$-strong Cauchy. This implies, because of Proposition 7, that the dynamics for the physical system can be obtained as a $\tau$-limit of the regularized dynamics $x_{\alpha}(t)$, which is a fixed point of $\lim _{\alpha} U_{\alpha}$.

4. Analysis of the dynamics at the infinitesimal level. In this section we discuss the dynamics of a physical system considering the case in which no hamiltonian exists for the system itself. We will only assume that there exists a family of regularized energy operators, $\left\{H_{L}\right\}$, one for each value of the cutoff $L$.

Let $\left(\mathcal{A}, \mathcal{A}_{0}\right)$ be a quasi *-algebra.

Definition. A ${ }^{*}$-derivation of $\mathcal{A}_{0}$ is a map $\delta: \mathcal{A}_{0} \rightarrow \mathcal{A}$ with the following properties:

(i) $\delta\left(x^{*}\right)=\delta(x)^{*}, \forall x \in \mathcal{A}_{0}$; 
(ii) $\delta(\alpha x+\beta y)=\alpha \delta(x)+\beta \delta(y), \forall x, y \in \mathcal{A}_{0}, \forall \alpha, \beta \in \mathbb{C}$;

(iii) $\delta(x y)=x \delta(y)+\delta(x) y, \forall x, y \in \mathcal{A}_{0}$.

Definition. Let $\left(\mathcal{A}, \mathcal{A}_{0}\right)$ be a quasi ${ }^{*}$-algebra, $\mathcal{D}_{\pi}$ a dense domain in a certain Hilbert space $\mathcal{H}_{\pi}$, and $\pi$ a linear map from $\mathcal{A}$ into $\mathcal{L}\left(\mathcal{D}_{\pi}, \mathcal{H}_{\pi}\right)$ such that:

(i) $\pi\left(a^{*}\right)=\pi(a)^{\dagger}, \quad \forall a \in \mathcal{A}$;

(ii) if $a \in \mathcal{A}, x \in \mathcal{A}_{0}$, then $\pi(a) \bullet \pi(x)$ is well defined and $\pi(a x)=\pi(a) \bullet \pi(x)$.

Here $\bullet$ is the weak multiplication defined in partial *-algebras, [AK]. We say that such a map $\pi$ is a ${ }^{*}$-representation of $\mathcal{A}$. Moreover, if

(iii) $\pi\left(\mathcal{A}_{0}\right) \subset \mathcal{L}^{\dagger}\left(\mathcal{D}_{\pi}\right)$

then $\pi$ is a ${ }^{*}$-representation of the quasi *algebra $\left(\mathcal{A}, \mathcal{A}_{0}\right)$.

Let $\pi$ be a ${ }^{*}$-representation of $\left(\mathcal{A}, \mathcal{A}_{0}\right)$. The strong topology $\tau_{s}$ on $\pi(\mathcal{A})$ is the locally convex topology defined by the following family of seminorms: $\left\{p_{\xi}(.) ; \xi \in \mathcal{D}_{\pi}\right\}$, with $p_{\xi}(\pi(a)) \equiv\|\pi(a) \xi\|$, where $a \in \mathcal{A}, \xi \in \mathcal{D}_{\pi}$.

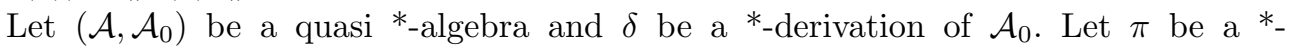
representation of $\left(\mathcal{A}, \mathcal{A}_{0}\right)$.

We will always assume that whenever for $x \in \mathcal{A}_{0} \pi(x)=0$, then $\pi(\delta(x))=0$.

Under this assumption, the linear map

$$
\delta_{\pi}(\pi(x))=\pi(\delta(x)), \quad x \in \mathcal{A}_{0},
$$

is well-defined on $\pi\left(\mathcal{A}_{0}\right)$ with values in $\pi(\mathcal{A})$ and it is a ${ }^{*}$-derivation of $\pi\left(\mathcal{A}_{0}\right)$. We call $\delta_{\pi}$ the *-derivation induced by $\pi$.

Given such a representation $\pi$ and its dense domain $\mathcal{D}_{\pi}$, we consider the usual graph topology $t_{\dagger}$ generated by the seminorms

$$
\xi \in \mathcal{D}_{\pi} \rightarrow\|A \xi\|, \quad A \in \mathcal{L}^{\dagger}(\mathcal{D}) .
$$

It is also easy to produce a representation on a quasi *-algebra. Calling $\mathcal{D}_{\pi}^{\prime}$ the conjugate dual of $\mathcal{D}_{\pi}$ we get the usual rigged Hilbert space $\mathcal{D}_{\pi}\left[t_{\dagger}\right] \subset \mathcal{H}_{\pi} \subset \mathcal{D}_{\pi}^{\prime}\left[t_{\dagger}^{\prime}\right]$, where $t_{\dagger}^{\prime}$ denotes the strong dual topology of $\mathcal{D}_{\pi}^{\prime}$. As usual we introduce $\mathcal{L}\left(\mathcal{D}_{\pi}, \mathcal{D}_{\pi}^{\prime}\right)$ and $\mathcal{L}^{\dagger}\left(\mathcal{D}_{\pi}\right)$. In this case, $\mathcal{L}^{\dagger}\left(\mathcal{D}_{\pi}\right) \subset \mathcal{L}\left(\mathcal{D}_{\pi}, \mathcal{D}_{\pi}^{\prime}\right)$. We know that each operator $A \in \mathcal{L}^{\dagger}\left(\mathcal{D}_{\pi}\right)$ can be extended to all of $\mathcal{D}_{\pi}^{\prime}$ in the following way:

$$
\left\langle\hat{A} \xi^{\prime}, \eta\right\rangle=\left\langle\xi^{\prime}, A^{\dagger} \eta\right\rangle, \quad \forall \xi^{\prime} \in \mathcal{D}_{\pi}^{\prime}, \quad \eta \in \mathcal{D}_{\pi} .
$$

Therefore the multiplication of $X \in \mathcal{L}\left(\mathcal{D}_{\pi}, \mathcal{D}_{\pi}^{\prime}\right)$ and $A \in \mathcal{L}^{\dagger}\left(\mathcal{D}_{\pi}\right)$ can always be defined:

$$
(X \circ A) \xi=X(A \xi) \text {, and }(A \circ X) \xi=\hat{A}(X \xi), \quad \forall \xi \in \mathcal{D}_{\pi} .
$$

With these definitions it is known that $\left(\mathcal{L}\left(\mathcal{D}_{\pi}, \mathcal{D}_{\pi}^{\prime}\right), \mathcal{L}^{\dagger}\left(\mathcal{D}_{\pi}\right)\right)$ is a quasi *-algebra, and $\pi$ represents $\left(\mathcal{A}, \mathcal{A}_{0}\right)$ into $\left(\mathcal{L}\left(\mathcal{D}_{\pi}, \mathcal{D}_{\pi}^{\prime}\right), \mathcal{L}^{\dagger}\left(\mathcal{D}_{\pi}\right)\right)$.

In $[\mathrm{BIT}]$ we have proved the following

TheOREM 8. Let $\left(\mathcal{A}, \mathcal{A}_{0}\right)$ be a locally convex quasi *algebra with identity $\mathcal{I}$ and $\delta$ be a *-derivation of $\mathcal{A}_{0}$. Then the following statements are equivalent:

(i) There exists a $\left(\tau-\tau_{s}\right)$-continuous, ultra-cyclic * ${ }^{*}$-representation $\pi$ of $\mathcal{A}$, with ultracyclic vector $\xi_{0}$, such that the ${ }^{*}$-derivation $\delta_{\pi}$ induced by $\pi$ is spatial, i.e. there exists 
$H=H^{\dagger} \in \mathcal{L}\left(\mathcal{D}_{\pi}, \mathcal{D}_{\pi}^{\prime}\right)$ such that $H \xi_{0} \in \mathcal{H}_{\pi}$ and

$$
\delta_{\pi}(\pi(x))=i\{H \circ \pi(x)-\pi(x) \circ H\}, \quad \forall x \in \mathcal{A}_{0} .
$$

(ii) There exists a positive linear functional $f$ on $\mathcal{A}_{0}$ such that

$$
f\left(x^{*} x\right) \leq p(x)^{2}, \quad \forall x \in \mathcal{A}_{0},
$$

for some continuous seminorm $p$ of $\tau$ and, denoting by $\tilde{f}$ the continuous extension of $f$ to $\mathcal{A}$, the following inequality holds:

$$
|\tilde{f}(\delta(x))| \leq C\left(\sqrt{f\left(x^{*} x\right)}+\sqrt{f\left(x x^{*}\right)}\right), \quad \forall x \in \mathcal{A}_{0},
$$

for some positive constant $C$.

(iii) There exists a positive sesquilinear form $\varphi$ on $\mathcal{A} \times \mathcal{A}$ such that $\varphi$ is invariant, i.e.

$$
\varphi(a x, y)=\varphi\left(x, a^{*} y\right), \text { for all } a \in \mathcal{A} \text { and } x, y \in \mathcal{A}_{0}
$$

$\varphi$ is $\tau$-continuous, i.e.

$$
|\varphi(a, b)| \leq p(a) p(b), \text { for all } a, b \in \mathcal{A},
$$

for some continuous seminorm $p$ of $\tau$; and $\varphi$ satisfies the following inequality:

$$
|\varphi(\delta(x), \mathcal{I})| \leq C\left(\sqrt{\varphi(x, x)}+\sqrt{\varphi\left(x^{*}, x^{*}\right)}\right), \quad \forall x \in \mathcal{A}_{0},
$$

for some positive constant $C$.

In order to apply this result to $Q M_{\infty}$ we assume that there exists a $\left(\tau-\tau_{s}\right)$-continuous *-representation $\pi$ in the Hilbert space $\mathcal{H}_{\pi}$, which is ultra-cyclic with ultra-cyclic vector $\xi_{0}$, and a family of ${ }^{*}$-derivations $\left\{\delta_{n}: n \in \mathbb{N}\right\}$ of a locally convex quasi ${ }^{*}$-algebra $\left(\mathcal{A}, \mathcal{A}_{0}\right)$ with identity. We define a related family of $*_{\text {-derivations }} \delta_{\pi}^{(n)}$ induced by $\pi$ defined on $\pi\left(\mathcal{A}_{0}\right)$ and with values in $\pi(\mathcal{A})$ :

$$
\delta_{\pi}^{(n)}(\pi(x))=\pi\left(\delta_{n}(x)\right), \quad x \in \mathcal{A}_{0} .
$$

In $[\mathrm{BIT}]$ we have also proven the following

Proposition 9. Suppose that:

(i) $\left\{\delta_{n}(x)\right\}$ is $\tau$-Cauchy for all $x \in \mathcal{A}_{0}$;

(ii) For each $n \in \mathbb{N}, \delta_{\pi}^{(n)}$ is spatial, that is, there exists an operator $H_{n}$ such that

$$
\begin{gathered}
H_{n}=H_{n}^{\dagger} \in \mathcal{L}\left(\mathcal{D}_{\pi}, \mathcal{D}_{\pi}^{\prime}\right), \\
H_{n} \xi_{0} \in \mathcal{H}_{\pi} \text { and } \delta_{\pi}^{(n)}(\pi(x))=i\left\{H_{n} \circ \pi(x)-\pi(x) \circ H_{n}\right\}, \forall x \in \mathcal{A}_{0} ;
\end{gathered}
$$

$$
\sup _{n}\left\|H_{n} \xi_{0}\right\|=: L<\infty \text {. }
$$

Then we have:

(a) $\exists \delta(x)=\tau-\lim \delta_{n}(x)$, for all $x \in \mathcal{A}_{0}$, which is a ${ }^{*}$-derivation of $\mathcal{A}_{0}$;

(b) $\delta_{\pi}$, the ${ }^{*}$-derivation induced by $\pi$, is well-defined and spatial; 
(c) if $H$ is the self-adjoint operator which implements $\delta_{\pi}$, if $<\left(H_{n}-H\right) \xi_{0}, \xi>\rightarrow 0$ for all $\xi \in D_{\pi}$ then $H_{n}$ converges weakly to $H$.

This result can be seen in the following way: even if the sequence $\left\{H_{n}\right\}$ of operators which implement $\left\{\delta_{n}\right\}$ does not converge, the sequence $\delta_{n}(X)$ may converge in the topol$\operatorname{ogy} \tau$. This is what happens, for instance, to mean field spin systems, when $X$ is a local operator, $[\mathrm{BM}]$. Therefore, this limit defines a derivation $\delta$ which, under the assumptions of Proposition 9, is spatial. This means that an effective hamiltonian $H_{\pi}^{e f f}$, in the sense of [BT4], can be defined, and this simplifies the successive analysis of the finite time evolution of the system $\mathcal{S}$. We recall here that a model admits an effective hamiltonian in the ${ }^{*}$-representation $\pi$ in the sense of [BT4] if there exists a self-adjoint operator $H_{\pi}^{\text {eff }}$ in $\mathcal{H}_{\pi}\left(\supset \mathcal{D}_{\pi}\right)$ with the property

$$
\pi(\delta(A))=i\left[H_{\pi}^{e f f}, \pi(A)\right], \quad \forall A \in \mathcal{A}_{0} .
$$

This equation is understood in the following weak sense:

$$
<\pi(\delta(A)) \phi, \psi>=i\left\{<\pi(A) \phi, H_{\pi}^{e f f} \psi>-<H_{\pi}^{e f f} \phi, \pi\left(A^{*}\right) \psi>\right\},
$$

for all $\phi, \psi \in D\left(H_{\pi}^{e f f}\right)$ and for all $A \in \mathcal{A}_{0}$.

The conclusion we get, therefore, is that Proposition 9 produces a sufficient condition for a model to admit an effective hamiltonian, so that all the results given in [BT4] can be adapted to this situation.

\section{References}

[AK] J.-P. Antoine and W. Karwowski, Partial *-algebras of closed linear operators in Hilbert space, Publ. RIMS, Kyoto Univ. 21 (1985), 205-236.

[B1] F. Bagarello, Applications of topological *algebras of unbounded operators, J. Math. Phys. 39 (1998), 2730-2747.

[B2] F. Bagarello, Fixed points in topological *-algebras of unbounded operators, Publ. RIMS, Kyoto Univ. 37, (2001), 397-418.

[B3] F. Bagarello, Relations between the Hepp-Lieb and the Alli-Sewell laser models, Ann. H. Poincaré 3 (2002), 983-1002.

[B4] F. Bagarello, The stochastic limit in the analysis of the BCS model, J. Phys. A 37 (2004), $2537-2548$.

[BIT] F. Bagarello, A. Inoue and C. Trapani, Derivations of quasi *-algebras, Int. Journ. Math. and Math. Sci. 21 (2004), 1077-1096.

[BM] F. Bagarello and G. Morchio, Dynamics of mean-field spin models from basic results in abstract differential equations, J. Stat. Phys. 66 (1992), 849-866.

[BR] O. Bratteli and D. W. Robinson, Operator Algebras and Quantum Statistical Mechanics 2, Springer-Verlag, New York, 1987.

[BS] F. Bagarello and G. L. Sewell, New structures in the theory of the laser model II: microscopic dynamics and a non-equilibrium entropy principle, J. Math. Phys. 39 (1998), 2730-2747.

[BT1] F. Bagarello and C. Trapani, 'Almost' mean field Ising model: an algebraic approach, J. Statistical Phys. 65 (1991), 469-482. 
[BT2] F. Bagarello and C. Trapani, A note on the algebraic approach to the "almost" mean field Heisenberg model, Il Nuovo Cimento B 108 (1993), 779-784.

[BT3] F. Bagarello and C. Trapani, States and representations of $C Q^{*}$-algebras, Ann. Inst. H. Poincaré 61 (1994), 103-133.

[BT4] F. Bagarello and C. Trapani, The Heisenberg dynamics of spin sistems: a quasi*-algebras approach, J. Math. Phys. 37 (1996), 4219-4234.

[BT5] F. Bagarello and C. Trapani, Algebraic dynamics in $O^{*}$-algebras: a perturbative approach, J. Math. Phys. 43 (2002), 3280-3292.

[DS] D. A. Dubin and G. L. Sewell, Time-translations in the algebraic formulation of statistical mechanics, J. Math. Phys. 11 (1970), 2990-2998.

[HK] R. Haag and D. Kastler, An algebraic approach to quantum field theory, J. Math. Phys. 5 (1964), 848-861.

[L] G. Lassner, Topological algebras and their applications in quantum statistics, Wiss. Z. KMU-Leipzig, Math.-Naturwiss. R. 30 (1981), 572-595.

[MS] G. Morchio and F. Strocchi, Mathematical structures for long range dynamics and symmetry breaking, J. Math. Phys. 28 (1987), 622-635.

[S] S. Sakai, Operator Algebras in Dynamical Systems, Cambridge Univ. Press, Cambridge, 1991.

[TW] W. Thirring and A. Wehrl, On the mathematical structure of the B.C.S.-model, Commun. Math. Phys. 4 (1967), 303-314.

[Tr] C. Trapani, Quasi *-algebras of operators and their applications, Reviews Math. Phys. 7 (1995), 1303-1332. 